\title{
Further studies on the genetic heterogeneity of cebocephaly
}

\author{
G. I. LAZJUK, I. W. LURIE, and M. K. NEDZVED \\ Laboratory of Teratology and Medical Genetics, Minsk Medical Institute, Minsk, USSR
}

Summary. The detailed morphological description of 4 cases with cebocephaly, 3 of which were karyotyped (one with D trisomy and 2 with normal karyotypes), are presented. Analysis of all cytogenetically studied cases with this malformation reveals that cebocephaly with a normal karyotype may result from more than one mutant gene, and so it may be accompanied by different extracranial abnormalities. On the other hand an absence of visceral malformations does not exclude chromosomal aberrations; thus $18 \mathrm{p}$ - syndrome, where cebocephaly is frequent, may have no visceral abnormalities.

Recently Holmes, Driscoll, and Atkins (1974) reported cytogenetic and pathomorphological investigations in 3 cases of cebocephaly and reviewed previous cytogenetic observations of this malformation. Four of 13 children had a normal karyotype, 4 had trisomy 13,2 had 18p- deletion, 1 had $r(18)$, 1 had trisomy $\mathrm{C}$, and 1 had trisomy 18 . In addition Yanoff, Rorke, and Niederer (1970) and Schinzel et al (1974) reported cebocephaly in children with loss of a short arm of chromosome 18 as a result of a $r(18)$ and $t(D q 18 q)$, respectively.

The analysis of morphological data in cebocephaly enabled Holmes et al (1974) to conclude that cebocephaly with a normal karyotype is an isolated malformation or is combined with only a few extracranial anomalies. On the other hand, a complex of extracranial malformations, including visceral ones, is typical of cebocephaly with an abnormal karyotype. However, in our opinion cebocephaly with a normal karyotype is also genetically heterogeneous.

During the past 6 years (1969 to 1974) we have seen 4 cases of cebocephaly, 3 of which were karyotyped. In one case cytogenetic study was unsuccessful. We present here descriptions of these cases.

\section{Case reports}

Case 1 (DI 140573). This female infant was born to a gravida-4, 41-year-old, obese mother and a 34-year-

Received 21 August 1975. old father with cryptorchidism. The first pregnancy of the mother in 1967 was complicated by a threatened abortion during the first trimester, and the child, a boy, died at the age of 1 month from pneumonia. The second and third pregnancies (both in 1971) terminated in spontaneous abortions at 8 and 12 weeks of gestation. The fourth pregnancy was complicated by polyhydramnios. The infant was delivered at 39 weeks, but she developed severe asphyxia and died within an hour.

Postmortem clinical examination. Weight $2460 \mathrm{~g}$, head circumference $25 \mathrm{~cm}$. The head was small with a slanting forehead and occiput; mild hypotelorism with canthal index $30.5 \%$ (normal for age-38\%), a tubular soft nose with a single opening (Fig. 1a), 3 round scalp defects in the parietal area, bilateral microphthalmia, and typical key-like colobomas of the iris were also present. The examination also revealed microretrognathy, dysplastic ears with underdeveloped helix, bilateral ulnar postaxial polydactyly, and rudimentary additional toes bilaterally.

The brain was underweight, $94 \mathrm{~g}$ (normal $308 \mathrm{~g}$ ). The telencephalon was not divided into hemispheres and there was a single cerebral ventricle covered with pia mater (Fig. 2a). The corpus callosum, olfactory bulbs, tracts, and nerves were absent. The gyri of the telencephalon were broad and disorientated. Thalami and caudate nuclei were not divided, and the third ventricle was absent. Pons, hippocampus, and cerebellar vermis were hypoplastic; Ammon's horns were not found. The olives were large; the pyramids of the medulla oblongata were not identified.

The internal base of the cranium was deformed, the anterior and the middle cranial fossae being more 314 

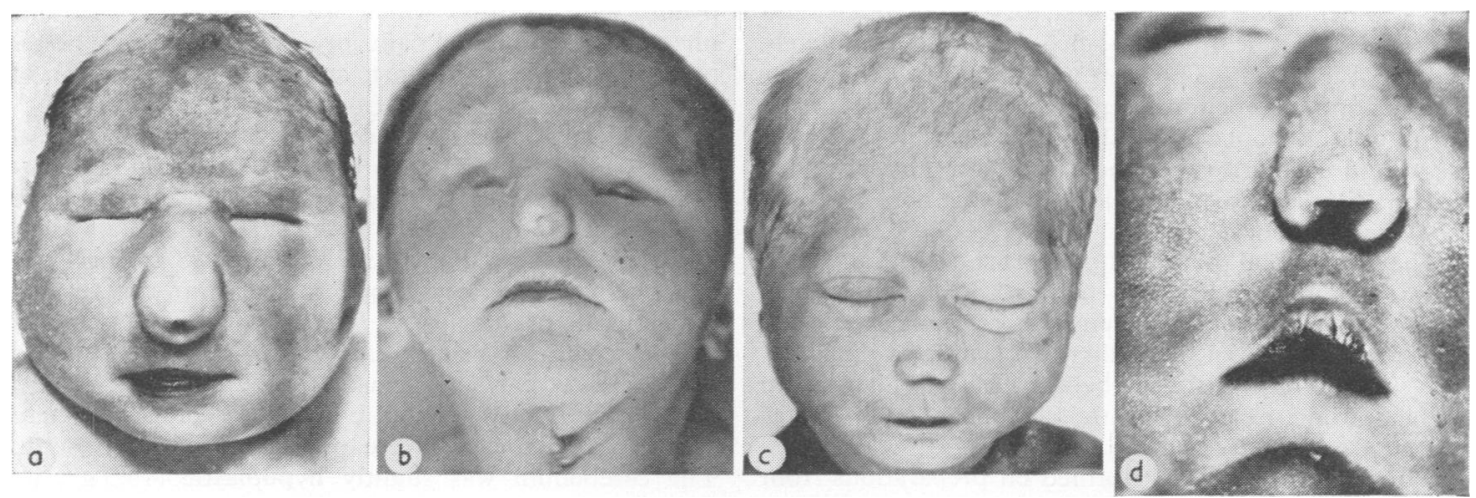

Fig. 1. Cebocephaly. (a) Case 1; (b) Case 2; (c) Case 3; (d) Case 4.
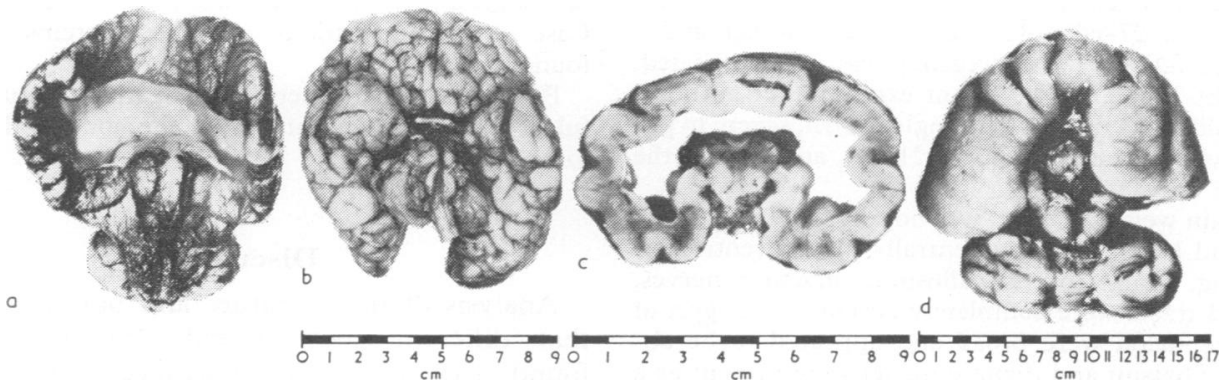

FIG. 2. The brain in cebocephaly. (a) Alobar holoprosencephaly in Case 1; (b) arrhinencephaly in Case 2; (c) frontal section of a brain in Case 3, with single venticular cavity; (d) failure of division of the forebrain in Case 4.

shallow than usual. Crista galli was absent, the lamina cribrosa being deformed.

Necropsy also showed a spurious diaphragmatic hernia on the left with intrusion of the spleen, left part of the liver, intestinal and colonic loops into the pleural cavity. The lungs weighed only $11.5 \mathrm{~g}$ (expected weight-50 g). There was also transposition of the large vessels of the heart, excessively lobulated kidneys with many small cysts, an incompletely rotated colon, with a doublebarrel-like sigma, an ectopic spleen and heterotopy of the splenic tissue into the pancreas, and a bicornuate uterus.

Microscopical examination of the brain showed an absence of normal stratification of neurones in all parts of the telencephalon, differentiated neurones being located on different levels. Ammon's horns and some nuclear groups in the thalamus were not identified. The pyramids were severely hypoplastic. There were clusters of heterotopic cells of the embryonal layer of cerebellar cortex and Purkinje-cells in the putamen, and many clusters of heterotopic cells of the embryonal layer were found in the dentate nuclei.

Cytogenetic investigation was performed on lymphocytes obtained at necropsy. Chromosome analysis showed D-trisomy in all suitable cells. Karyotype: $47, \mathrm{XX},+\mathrm{D}$.
Case 2 (FD 010971). A male born to a gravida-6, 40-year-old mother and 36-year-old father. First (1957) and fourth (1965) pregnancies had resulted in normal children (boy and girl). Therapeutic abortions as a result of severe toxaemia were performed in the second (1960) and fifth (1969) pregnancies. After an abortion in 1960 she suffered from adnexitis and received treatment in hospital and as an outpatient. The third pregancy (1964) had resulted in a spontaneous abortion.

In the second week of the pregnancy the mother had an attack of respiratory distress with fever and took antibiotics and sulphonamides. The pregnancy was complicated by toxaemia, vaginal bleeding in the first and second trimesters, and polyhydramnios (7 litres). Delivery took place at term, birthweight $2700 \mathrm{~g}$. The baby lived 3 hours before dying in respiratory distress.

Necropsy revealed antimongoloid slanting of the eyes, bilateral anophthalmia, a proboscis-like nose without a nostril (Fig. 1b), microretrognathy, prominent occiput, craniostenosis, additional skin tags near the ears, low hair line on the neck, aplasia of the tricuspid valve, hyperplasia of the thymus, weight $20 \mathrm{~g}$ (vs. normal $10 \mathrm{~g}$ ), excess of embryonal lobulation, and cortical cysts in the kidneys.

The brain weighed $362 \mathrm{~g}$ (normal) and was divided 
into two hemispheres. The olfactory bulbs, tracts, and trigones, optic nerves, and chiasma were completely absent (Fig. 2b). The corpus callosum, ventricular system, and cerebellum appeared normal. Ammon's horns were hypoplastic. The cranium showed hyperplasia and a deformity of the crista galli, aplasia of the lamina cribrosa and an additional metopic bone between the 2 frontal bones.

The histological picture of the cortex cerebri and subcortical nuclear groups was normal. Many immature cells were found around ventricles. Some dysplastic gyri from the cells of the embryonal layer and Purkinjecells were found in the cerebellar vermis. There were no heterotopic neurones in the cerebellar white matter and nuclear groups.

Cytogenetic analysis performed on preparations from lymphocyte culture obtained at necropsy revealed a normal male karyotype-46,XY.

Case 3 (PN 110273). A boy born at 37 weeks' gestation to a 27-year-old primigravida mother and a 26-year-old father. The pregancy was uncomplicated, birthweight $2600 \mathrm{~g}$. The infant expired after an hour.

The child had a nose with a single nostril, severe hypotelorism with a canthal index of $21.4 \%$, and cleft of the soft palate (Fig. 1c).

The brain weighed $194 \mathrm{~g}$, was not divided into hemispheres, and had a common centrally placed ventricular cavity (Fig. 2c). Corpus callosum, olfactory nerves, bulbs, and tracts were completely absent. The gyri of the telencephalon were broad and appeared to be abnormal. Thalami and dentate nuclei were present as a single body. The pons and the hippocampus were slightly hypoplastic. The medulla oblongata had large olives and severely hypoplastic pyramids. The cerebellum appeared normal.

The base of the skull was similar to that of Case 1 . Normal stratification of the cortex in all regions was distorted. There were insufficiently differentiated neurones among the mature ones. Ammon's horns were severely hypoplastic, but followed a normal course. The fields of Ammon's horns and the nuclear groups of the thalamus were not distinguishable. The pyramids showed severe hypoplasia. The cerebellar cortex appeared normal and heterotopic cells in cerebellar white matter and its nuclear groups were not found. There were no extracranial malformations in this case.

Cytogenetic examination was performed on the cultured skin fibroblasts obtained at necropsy. A normal male karyotype $46, \mathrm{XY}$ was established in all suitable metaphases.

Case 4 (AZ 190569). A female, the third-born child of a 41-year-old, gravida-5 mother and a 43-year-old father. Two female sibs are living and well. The 3rd and 4th pregnancies terminated in medical abortions. After the second abortion the mother suffered from adnexitis and was treated by antibiotics and other preparations. She works as a fitter at a radio factory and has contact with lead and tin.

The pregnancy was complicated at 5 to 6 weeks by pyelitis and cystitis, which were treated by chloramphenicol. Abdominal pains occurred throughout gesta- $\vec{D}$

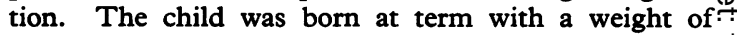
$2700 \mathrm{~g}$ but died at 1 hour from severe asphyxia.

There was a peculiar face with a blind-ended nasal $\stackrel{\text { ? }}{\rightarrow}$ orifice (Fig. 1d), hypotelorism, typical bilateral colo-읃 bomas of the iris, narrow palpebral fissures, cleft of the hard palate, and deformity of the external ears.

Necropsy showed a brain weighing $88 \mathrm{~g}$ (Fig. 2d). The hemispheres were not divided in the fronto-

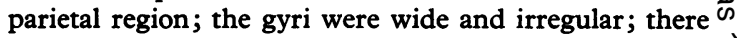
was a common ventricular cavity; and the corpus $\overrightarrow{0}$ callosum was partially aplastic. The olfactory nerves, $\vec{\overrightarrow{ }}$ bulbs, and tracts, and optic chiasma were completely $\vec{\omega}$ absent. The thalami were present as a single body. The cerebellum was slightly hypoplastic $(15.5 \mathrm{~g})$; its vermis was small, wide, and flat. The dentate nuclei were hardly defined. The medullary olives were $\vec{\omega}$ enlarged. The internal base of the skull and the histological picture of the brain were similar to those of $\vec{\omega}$ Case 3. Malformations in other systems were not found.

Postmortem cytogenetic investigation was unsuccess- ful because of late admission of the body to the labora- $\square$ tory.

\section{Discussion}

Analysis of the literature and our data indicate that different chromosomal abnormalities were found in 12 out of 18 cases with cebocephaly, where karyotype analysis was performed (Yanoff et al, 1970; Holmes et al, 1974; Schinzel et al, 1974; our data), and in 6 cases the karyotype was normal. This probably does not represent the true frequency $\overrightarrow{0}$ of chromosome abnormalities in cases of cebo- $\exists$ cephaly in neonates since there is a good reason to think that cases of cebocephaly with an abnormal karyotype are more likely to be published. In only two instances (Holmes et al, 1974, and our work) was cebocephaly rather than the chromosomal aberration the initial premise for the presentation of the cases. In these reports there were 3 cases with normal karyotypes and 3 with abnormal ones.

Cebocephaly with an abnormal karyotype can be 옥 divided into 2 main groups: (i) cebocephaly as a $>$ trait to different malformations, (ii) cebocephaly as unique malformation (Lejeune et al, 1969; Holmes $N$ et al, 1974).

There are two types of abnormalities (trisomy 13 N and loss of the short arm of chromosomes 18) where $\omega$ cebocephaly is a typical feature. There are 5 cases of cebocephaly with trisomy 13 (Holmes et al, 1974;0 our Case 1). In all these cases this malformation $\mathbb{\Phi}$ was accompanied by holoprosencephaly and multiple $\stackrel{\text { ? }}{+}$ extracranial malformations typical of Patau's syn- $\frac{T}{0}$ drome. 
Cebocephaly with partial loss of the short arm of chromosome 18 has been described five times, with both 18p- deletion (Uchida et al, 1965; Gorlin, Yunis, and Anderson, 1968), and ring-18 chromosome (Yanoff et al, 1970; Neu et al, 1971) or $\mathrm{D} / 18$-translocation (Schinzel et al, 1974). Holoprosencephaly is also common in these cases, whereas extracranial malformations vary from case to case. Sometimes they are severe (Neu et al, 1972), while in other cases visceral abnormalities are slight (Uchida et al, 1965; Schinzel et al, 1974). Visceral malformations are not obligatory features in cases with loss of the short arm of chromosome 18 (Lurie and Lazjuk, 1972), so, in our mind, chromosomal abnormalities may be suspected even in cases without any extracranial abnormalities. Therefore, it is necessary to perform cytogenetic investigation in every case with cebocephaly.

The case of cebocephaly in trisomy 18 (Holmes et $a l, 1974)$ is the only one from more than 400 reported cases of this syndrome. The abnormalities are not typical of this syndrome, and there are good reasons to suggest that cebocephaly in Edwards' syndrome is coincidental in this case, though other possibilities are not excluded.

In the case of trisomy C (Lejeune et al, 1969) necropsy was not performed and the abnormal chromosome was not fully identified. In the light of new data (Dinno, Silvey, and Weisskopf, 1974) pure trisomy $\mathrm{C}$ cannot be considered as the only interpretation of this aberration.

Cebocephaly with a normal karyotype is also heterogeneous from the genetical point of view. In most cases it is virtually an isolated malformation with a typical morphological picture (de Myer, 1964; Case 1 of Holmes et al, 1974; our Case 3 and probably Case 4). Though teratogenic influences cannot be completely excluded the most likely cause of cebocephaly in such cases is homozygosity for an autosomal recessive mutant gene (James and van Leeuwen, 1970). In such cases it is accompanied usually by holoprosencephaly. However, in some cases cebocephaly may be a feature of nonchromosomal syndromes with multiple congenital anomalies (our Case 2) caused by genic mutation or by environmental stimuli. Such a possibility must always be borne in mind, and, therefore, the occurrence of multiple malformations in cases with cebocephaly cannot serve as conclusive evidence of chromosomal abnormality, but only an indication of its possibility.

From the morphological point of view it is necessary to take into account the fact that the combination of cebocephaly and prosencephaly is common, but not constant, and in some cases (e.g. our Case 2) the proboscis-like nose may occur with arrhinencephaly, the brain being divided into hemispheres. Though on the whole there is a distinct correlation between abnormalities of the face and the brain (de Myer, Zeman, and Palmer, 1964), exceptions, similar to our Case 2, have already been reported (Patel, Dolman, and Byrne, 1972).

From the point of view of formal genesis cebocephaly is a result of misdivision of the prosencephalon. In cases with chromosomal abnormalities it is caused probably by the delay of normal development resulting from an excess of genetic information or from its arrest because of a lack of chromosomal material. The assumption about the hemizygosity of a recessive gene on the intact homologue suggested first by Uchida et al (1965) is unlikely to be true, since arrhinencephaly is too frequently associated with the loss of the short arm of chromosome 18.

Division of the prosencephalon ends early, about the 30th day of embryonal development (Yakovlev, 1959). If the prosencephalon is not normally divided, both the telencephalon and the diencephalon are involved and that will lead to the nondivision of thalami optici and dysgenesis of the pituitary gland. Relative independence of division of the telencephalon from the processes of differentiation of the mesencephalon will lead to a condition in which cebocephaly is accompanied by alobar holoprosencephaly, in some cases by a semilobar one, and by arrhinencephaly with complete division of telencephalon into hemispheres. Relative discorrelations between the development of the brain and the nasal structures, as in our Case 2, most probably point to a local injury of some process in normal embryogenesis by a teratogen. In our case the damage may be influenced by infection at the beginning of pregnancy or by the drugs used for treatment.

Thus, cebocephaly is a more heterogeneous condition than was previousy considered. It may be the result of chromosomal aberrations, gene mutation, and possibly of various environmental events. In cases with karyotype abnormalities, cebocephaly is accompanied usually by a complex of visceral malformations typical of this chromosomal syndrome. Since extracranial abnormalities in $18 \mathrm{p}-$ syndrome are not obligatory features, chromosomal abnormalities should not be excluded in the absence of visceral pathology. On the other hand multiple congenital abnormalities accompanied by cebocephaly may be the result of Mendelian genes or of environmental events; therefore the discovery of visceral abnormalities is not inconsistent with a normal karyotype in the child. 
A precise histological study of the brain can facilitate the diagnosis to a considerable extent. The absence of heterotopic cells of the embryonal layer and Purkinje-cells in the cerebellar white matter and of cells of the embryonal layer in the dentate nuclei is common for 'isolated' cebocephaly, whereas these abnormalities are constant features of Patau's syndrome.

Data on the histological structure of the cerebellum in cases resulting from the loss of the short arm of chromosome 18 are virtually absent, and the changes presented in the case by Gorlin et al (1968) are not specific. Therefore, it is not possible to judge whether or not there are morphological distinctions between 'isolated' cebocephaly and those resulting from $18 \mathrm{p}-$.

Undoubtedly, the type of morphological changes in the cerebrum is only a tentative test in the evaluation of every case, and a final conclusion can be made only after cytogenetic investigations.

\section{RBFERENCES}

De Myer, W. (1964). A 46 chromosome cebocephaly with remarks on the relation of 13-15 trisomy to holoprosencephaly (arrhinencephaly). Annales Paediatrici, 203, 169-177.

De Myer, W., Zeman, W., and Palmer, C. (1964). The face predicts the brain. Pediatrics, 34, 256-263.
Dinno, N. D., Silvey, G. L., and Wiesskopf, B. (1974). 47,XY, $t(9 p+; 11 q+)$ in a male infant with multiple malformations. Clinical Genetics, 6, 125-131.

Gorlin, R. J., Yunis, J., and Anderson, V. E. (1968). Short arm deletion of chromosome 18 in cebocephaly. American fournal of Diseases of Children, 115, 473-476.

Holmes, L. B., Driscoll, S., and Atkins, L. (1974). Genetic heterogeneity of cebocephaly. Fournal of Medical Genetics, 11, $35-40$.

James, E. and Leeuwen, G. van (1970). Familial cebocephaly. Case description and survey of the anomaly. Clinical Pediatrics, 9, 491-493.

Lejeune, J., Dutrillaux, B., Rethore, M. O., Berger, R., Debray, H., Veron, P., Gorce, F., and Grossiord, A. (1969). Sur trois cas de trisomie C. Annales de Génétique, 12, 28-35.

Lurie, I. W. and Lazjuk, G. I. (1972). Partial monosomies 18. Review of cytogenetical and clinical variants. Humangenetik, 15, 203-222.

Neu, R. L., Watanabe, N., Gardner, L. I., and Galvis, A. G. (1971). A single nasal orifice and severe intrauterine growth retardation in a case of 46,XX,18r. Annales de Génétique, 14, 139-142.

Patel, H., Dolman, C. L., and Byrne, M. A. (1972). Holoprosencephaly with median cleft lip. American fournal of Diseases of Children, 124, 217-221.

Schinzel, A., Schmid, W., Lüscher, U., Nater, M., Brook, C., and Steinmann, B. (1974). Structural aberrations of chromosome 18. I. The 18p-syndrome. Archiv für Genetik, 47, 1-15.

Uchida, I. A., McRae, K. N., Wang, H. C., and Ray M. (1965) Familial short arm deficiency of chromosome 18 concomitant with arhinencephaly and alopecia congenita. American fournal of Human Genetics, 17, 410-419.

Yakovlev, P. I. (1959). Pathoarchitectonic studies of cerebral malformations. III. Arrhinencephalies (holotelencephalies). fournal of Neuropathology and Experimental Neurology, 18, 22-55.

Yanoff, M., Rorke, L. B., and Niederer, B. S. (1970). Ocular and cerebral abnormalities in chromosome 18 deletion defect. American fournal of Opthalmology, 70, 391-402. 